\title{
A sustainable culture of tardigrades (Hypsibius dujardini) for astrobiological laboratory activities
}

\author{
Patricia G. Núñez ${ }^{1 *}$, Nayeli G. Quevedo ${ }^{2}$, y Roberto Vázquez ${ }^{3}$ \\ ${ }^{1}$ Instituto de Estudios Avanzados de Baja California, Ensenada, B.C. Mexico. \\ ${ }^{2}$ Facultad de Ciencias, Universidad Autónoma de Baja California, Ensenada, B.C. Mexico. \\ ${ }^{3}$ Laboratorio de Astrobiología, Instituto de Astronomía, Universidad Nacional Autónoma de México, \\ Ensenada, B.C. Mexico.
}

\begin{abstract}
Tardigrades are microscopic organisms that have gained importance in astrobiology in recent years. They have extreme survival ability and can enter into a state of latency for several years called cryptobiosis. Due to our interest in conducting experiments with tardigrades to complement the laboratory exercises with students in the course of astrobiology, we bought samples of tardigrades in the cryptobiotic state. A sample of these were cultured at room temperature $\left(18^{\circ} \mathrm{C}\right)$ with an oxygenation system, and fed with Spirulina algae every week. We found that in a few weeks tardigrades were producing eggs normally. This species, $H$. dujardini, has been cultured before and it is a good sample for culture as we saw. This species provides a good sustainable culture and is inexpensive and easy to produce tardigrades for didactic purposes.
\end{abstract}

\section{Introduction}

The Tardigrada phylum is a group of microscopic invertebrates, in which some of the species are found all over the world whereas other are endemic. They can be freshwater, marine or terrestrial $[1,2]$. They are generally known for the ability to withstand extreme environmental changes that other organisms could not (temperature, salinity, pressure, etc.), so they are classified as extremophile organisms. When unfavourable conditions appear in their environment, tardigrades are able to enter into a state of cryptobiosis, in which their metabolism is temporarily suspended and their body is compacted like a barrel. They have a cylindrical body with bilateral symmetry, four pairs of legs ending in nails or digits and a not well-defined head. Their form of reproduction is sexual or by parthenogenesis, and they have differentiated reproductive apparatus, indicating that they have sexual dimorphism [3].

Mature adults measure on average 250 to $500 \mu \mathrm{m}$, with very few species larger than $800 \mu \mathrm{m}$ (see Fig.1). Although some species exhibit colour in the intestine, storage cells, epidermis and/or cuticle, most are translucent or whitish (opaque), so their internal anatomy can be observed.

So far, more than 1200 species [4] have been arranged in two classes (Heterotardigrada and Eutardigrada).

In literature, some techniques are described which involve growing cultures of tardigrades, either in Erlenmeyer flasks or in petri dishes, adding Chalkey's medium and

\footnotetext{
Corresponding author: pgnunez@ideabc.org
} 
cultured algae $[5,6]$. Chalkeys medium is a solution that includes $\mathrm{CaCl} 2, \mathrm{NaCl}, \mathrm{KCl}$, and water [7].

\section{Objective}

To provide a new technique for cultivating tardigrades that will be more accessible for students and easy to maintain in a standard college laboratory, for Biology and Astrobiology experimental activities.

\section{Methodology}

A culture was done with tardigrade samples that were bought from Carolina Biological Supply Co. in cryptobiotic state (see Fig 2). Our method consists in the following steps. We used a small fishbowl, (Fig. 3), at room temperature $\left(18^{\circ} \mathrm{C}-22^{\circ} \mathrm{C}\right)$, in a low light condition, with $1200 \mathrm{ml}$ of fresh water. An aerated system with no filter was used. The culture was feed with 0.2 grams of Spirulina algae (Fig. 6) every week. Fresh water was added if necessary.

\section{Results and Discussion}

Hypsibius dujardini has been proposed to be a model of study for tardigrades [8], and is one of the most studied species, what makes it a good example for didactic purposes. The students could see live specimens of tardigrades in the course of Astrobiology 101 and could do some experiments to learn about their ability to survive in extreme conditions. The problem is that this is made in Petri dishes and should be filled with fresh water very often. This is to compensate for the evaporation that occurs as the day passes and the ambient temperature increases, because the higher the temperature, the greater the evaporation.

With our procedure there is less evaporation and we get good results. In a few weeks we had tardigrades out of the state of cryptobiosis producing eggs normally (Fig 5).. Also we observed a lot of juveniles (Fig. 7) and moults of different sizes (Fig. 4) when the culture is by old ( 7 months) we recommend to add more nutrients to the media like Chalkey's media, as a way to add nutrients to the culture.

This procedure provides a good sustainable culture and an inexpensive and easy method to produce tardigrades for didactic purposes.

\section{References}

1. Guil, N. and Cabrero-Sañudo, F. Biodiv. and Conservation, 16(4), pp.1063-1086 (2006)

2. McInnes S.J. Jour. Nat. Hist. , 28:2, 257-352 (1994)

3. Hummer, Paul J. Jr., The American Biology Teacher, Vol. 55 No. 6, Sep., 1993; (pp. 357-360)

4. Nelson, D., Guidetti, R. and Rebecchi, L.,) Ecology and classification of North American freshwater invertebrates. pp.455-484 (2010.

5. Gabriel, W. N., Mcnuff, R., Patel, S. K., Gregory, T., Jeck, W. R., Jones, C. D. And Goldstein, B. Develop. Biol. 312(2), pp. 545-559 (2007) 
6. Narváez P., Eliana X.; Jerez J., Javier H.; Biota Colombiana, noviembre, 145- 149 (2001)

7. Degma P. Bertolani R. and Guidetti R., Zootaxa, 1519, 41-53 (2017)

8. Gabriel, W.N; McNuff, R; Patel, S.K; et al. Dev. Biol. 312: 545-59. (December 2007)

\section{Acknowledgements}

This work has been supported by grants UNAM-DGAPA-PAPIME PE109915 and CONACYT-AEM 275311. Authors also thank the referee, Prof. Susana Deustua, for substantially improving this paper with her comments.

\section{Figures}

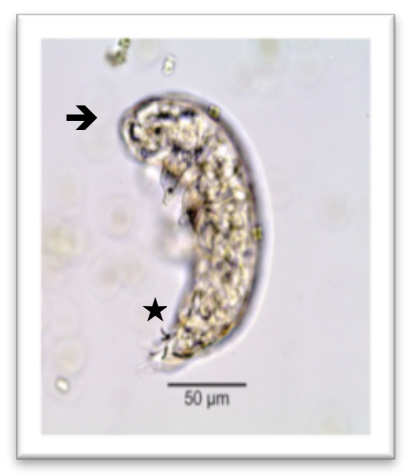

Fig. 1. Dorsal view Hypsibius dujardini, the head (black arrow), and the legs (star)

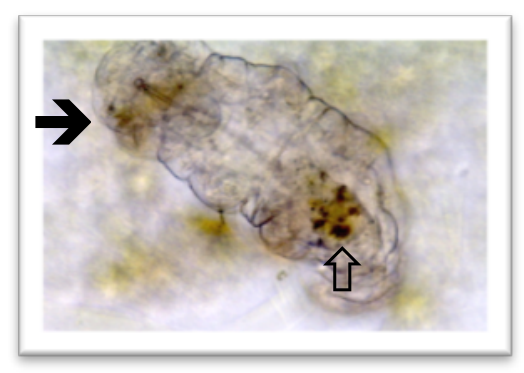

Fig. 2. Hypsibius dujardini in cryptobiosis state, head (black arrow) and digestive system (empty arrow). 


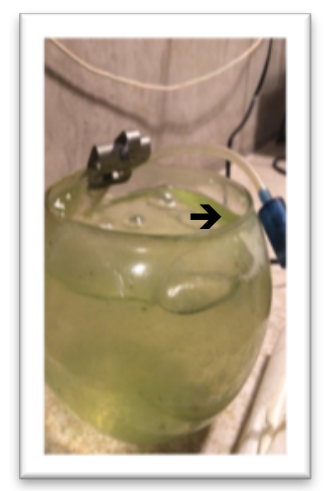

Fig. 3. Culture bowl with pump (aerated system).

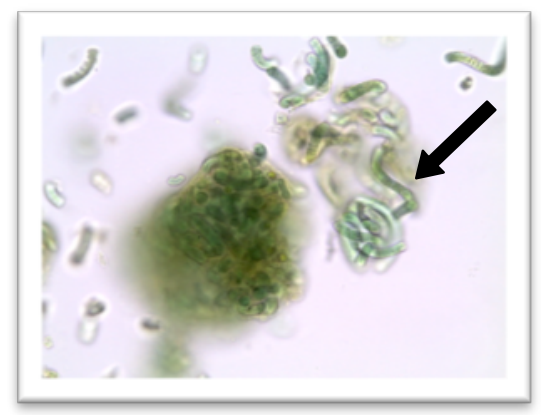

Fig. 4. Spirullina algae (black arrow).

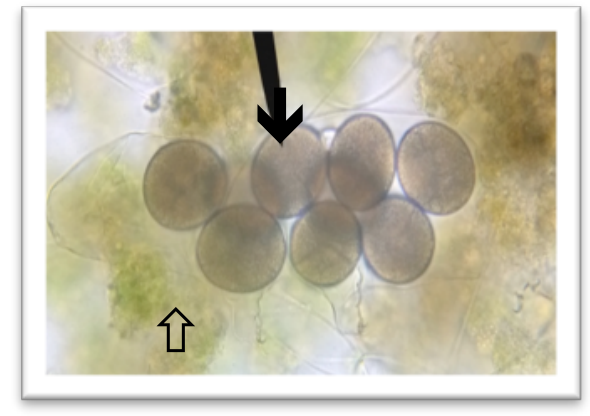

Fig. 5. Eggs inside moult (black arrow), and algae (empty arrow). 


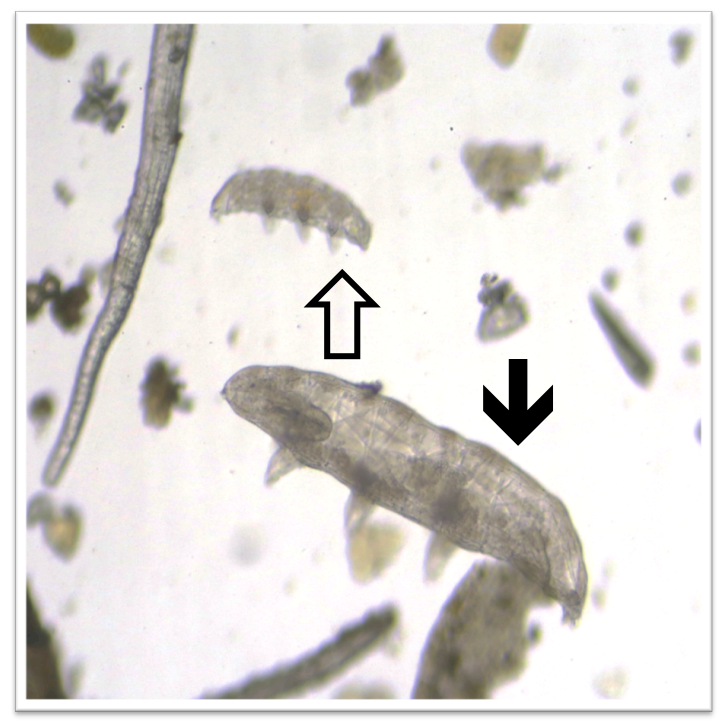

Fig. 6. Adult (black arrow) and juvenile tardigrades (empty arrow).

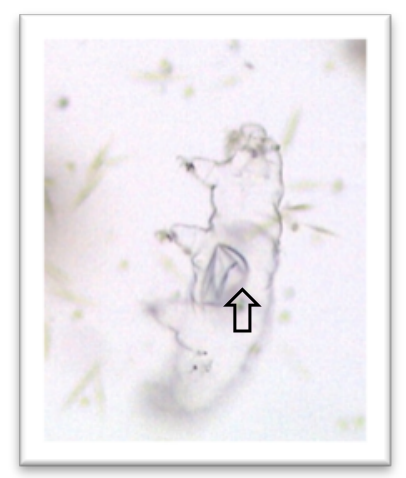

Fig. 7. Moult, with an empty egg (empty arrow). 